There is a growing body of literature examining potential hazards of engineered nanomaterials. This session will take stock of some of that literature and in particular, consider High Aspect Ratio Nanomaterials (HARNs) and more specifically carbon nanotubes. The first part of the session will address the oxidative/stress effects of various metallic nanoparticles. This will be followed by a discussion of a Horizon 2020 project known as BIORIMA. BIORIMA is a multinational collaboration to assess hazards of nano biomaterials and develop appropriate risk management tools. The remaining presentations will focus on a range of issues related to HARNs. These include assessing the similarities between carbon nanotubes (CNT) and asbestos, the identification of molecular initiating events of CNT in biological systems, the role of fibre rigidity in toxicity, mechanisms of CNT-induced inflammation, dustiness as a risk group strategy, the state of knowledge on occupational exposures, and risk management. Clearly there are many types of CNT in commerce and it is difficult to characterise the hazard with a single description. These sessions will help to identify the many factors that need to be considered.

\section{1b NONINVASIVE BIOMONITORING OF 3 GROUPS OF NANOMATERIALS WORKERS WITH ELEVATED MARKERS OF OXIDATIVE STRESS AND INFLAMMATION}

${ }^{1} \mathrm{D}$ Pelclova* ${ }^{2} \mathrm{~V}$ Zdimal, ${ }^{3} \mathrm{~S}$ Dvorackova, ${ }^{2} \mathrm{~S}$ Schwarz ${ }^{2} \mathrm{O}$ Ondracek, ${ }^{4} \mathrm{M}$ Komarc ${ }^{1} \mathrm{~S}$ VIckova, 'Z Fenclova, ${ }^{2} \mathrm{O}$ Makes, ${ }^{1} \mathrm{~S}$ Zakharov. ${ }^{1}$ Charles University and General University Hospital in Prague, First Faculty of Medicine, Department of Occupational Medicine, Prague, Czech Republic; ${ }^{2}$ Institute of Chemical Process Fundamentals of the CAS, Praque, Czech Republic; ${ }^{3}$ Technical University in Liberec, Faculty of Mechanical Engineering, Department of Machining and Assembly, Department of Engineering Technology, Department of Material Science, Liberec, Czech Republic; ${ }^{4}$ Charles University in Prague and General University Hospital in Prague, First Faculty of Medicine, Institute of Biophysics and Informatics, Prague, Czech Republic

\subsection{6/oemed-2018-ICOHabstracts.799}

Introduction The studies of workers exposed to nanomaterials are rather rare; however, the data are urgently needed, as the experimental data bring suspicion on deleterious effect by inhalation. The aim was to examine non-invasively workers exposed to varied materials, containing nanoparticles, and compare the results of the same markers of oxidation of lipids, nucleic acids and proteins, in addition to spirometry and fractional exhaled nitrogen oxide (FeNO).

Methods Three groups of workers were examined and compared with comparable control groups. They included:

- total 34 nanoTiO2 workers in two consecutive years

- 14 nano Fe oxides and

- total 32 nanocomposites workers in two consecutive years.

Aerosol exposure in nanocomposites was measured using offline and online aerosol instruments: Berner Low-Pressure Impactor, Scanning Mobility Particle Sizer, Aerodynamic Particle Sizer, Condensation Particle Counter and Optical Particle Sizer.

Following markers were analysed in the exhaled breath condensate (EBC) by liquid chromatography-electrospray ionization-tandem mass spectrometry (LC-ESI-MS/MS): malondialdehyde, 4-hydroxy-trans-hexenal, 4-hydroxy-trans-nonenal, 8isoProstaglandinF2 $\alpha$, C6-C13, 8-hydroxy-2-deoxyguanosine, 8hydroxyguanosine, 5-hydroxymethyl uracil, o-tyrosine, 3-chlorotyrosine, 3-nitrotyrosine and proinflammatory leukotrienes
LTB4, LTC4, LTE4, LTD4. Fractional exhaled nitric oxide (FeNO) and spirometry were measured.

Results The exposure to inert dusts exposure did not exceed allowed gravimetric limits for occupational exposure. However, all workers had elevated markers of oxidative stress, as compared to the controls. LTB4 and cysteinyl LTE4 were most involved inflammation markers. Most elevated markers were seen in nanoTiO2 workers, lower effect was seen both in the nano $\mathrm{Fe}$ oxides and nanocomposites exposed workers. On the other hand, spirometry and FeNO did not show any significant impairments.

Conclusions Non-invasive biomonitoring using markers of oxidative stress, LTB4 and LTE4 may be most useful and could be recommended biomarkers for preventive examinations and monitoring of workers with occupational exposure to nanoparticles.

Acknowledgement Progres Q25/1LF, Q296/1LF, 43/17/RPZP and CSFP503/12/G147.

\section{C THE POTENTIAL CARCINOGENICITY OF CARBON NANOTUBES (CNTS): SIMILARITIES AND DIFFERENCES WITH THE PATHOGENESIS OF ASBESTOS RELATED CANCERS}

H Wolff. Finnish Institute of Occupational Health, Helsinki, Finland

\subsection{6/oemed-2018-ICOHabstracts. 800}

Introduction Cancers and other diseases caused by asbestos and has led to the 'fibre paradigm' that in essence suggests that biopersistent, fibre like, materials have a potential for asbestos-like negative health effects. The overwhelmingly most common type of fiber-like nanomaterials are the carbon nanotubes (CNTs) of which there are numerous types. As predicted by the paradigm some CNTs have been shown to elicit tumour formation in animal models. Because of the very broad variety of CNTs it is imperative to understand which of these will have asbestos like effects. There are also unanswered questions about the carcinogenicity of CNTs that are not asbestos-like.

Methods The presentation will discuss asbestos related carcinogenicity and evaluate the differences and similarities to the current understanding of CNT related carcinogenesis. Special attention will be paid to in vivo studies and histology. In addition to discussing published literature some unpublished results will also be presented.

Results The early phases of asbestos induced effects in vivo include activation of inflammatory cascades and the production of reactive oxygen species. These effects are not that specific for asbestos and can be found in association with silica exposures and other exposures, including nanomaterials. A somewhat specific feature of asbestos exposure is a in vivo is eosinophilia associated with a Th2 type of immunological response. Interestingly this type of reaction can be seen in some types of CNTs with physical properties reminiscent of asbestos.

Discussion Some CNTs do have physicochemical properties and an in vivo inflammatory response similar to that of asbestos. The potential for carcinogenicity of CNTs without these asbestos-like features is rather poorly investigated. Additional studies with traditional animal models for carcinogenicity are needed to provide a foundation for other approaches to determine carcinogenic potential. 